\title{
OPEN Genetic basis and identification of candidate genes for wooden breast and white striping in commercial broiler chickens
}

\author{
Juniper A. Lake ${ }^{1,2}$, Jack C. M. Dekkers ${ }^{3} \&$ Behnam Abasht $^{1,2 \varpi}$
}

Wooden breast (WB) and white striping (WS) are highly prevalent and economically damaging muscle disorders of modern commercial broiler chickens characterized respectively by palpable firmness and fatty white striations running parallel to the muscle fiber. High feed efficiency and rapid growth, especially of the breast muscle, are believed to contribute to development of such muscle defects; however, their etiology remains poorly understood. To gain insight into the genetic basis of these myopathies, a genome-wide association study was conducted using a commercial crossbred broiler population $(n=1193)$. Heritability was estimated at 0.5 for WB and WS with high genetic correlation between them (0.88). GWAS revealed 28 quantitative trait loci (OTL) on five chromosomes for WB and 6 QTL on one chromosome for WS, with the majority of QTL for both myopathies located in $\mathrm{a} \sim 8 \mathrm{Mb}$ region of chromosome 5 . This region has highly conserved synteny with a portion of human chromosome 11 containing a cluster of imprinted genes associated with growth and metabolic disorders such as type 2 diabetes and Beckwith-Wiedemann syndrome. Candidate genes include potassium voltage-gated channel subfamily $Q$ member 1 (KCNO1), involved in insulin secretion and cardiac electrical activity, lymphocyte-specific protein 1 (LSP1), involved in inflammation and immune response.

The modern commercial broiler chicken embodies remarkable gains in the economics of meat production realized through intensive breeding programs, optimized nutrition, and enhanced management practices. Compared to the 1950s, modern broilers can be raised to approximately the same market weight in close to half the time using substantially less feed and with substantially higher breast muscle yield ${ }^{1-3}$. However, the financial gains and increased production capacity associated with improvements to production traits are threatened by the concurrent global emergence of numerous muscle disorders that severely affect meat quality and may also impact animal welfare ${ }^{4-6}$. Wooden breast and white striping, often co-occurring and believed to be part of the same disease spectrum ${ }^{7,8}$, are two such myopathies, which together represent the breast muscle defects with the highest prevalence and greatest economic burden.

First described in the literature in $2014^{4}$, wooden breast manifests as palpably firm and discolored pectoralis major with subcutaneous and fascial edema, petechial hemorrhages, and spongy areas with disintegrating myofiber bundles. Birds affected by wooden breast frequently show signs of white striping as well, which is macroscopically characterized by white fatty striations running parallel to the muscle fibers and presents with similar histological lesions as wooden breast, including myodegeneration with regeneration, necrosis, lymphocyte and macrophage infiltration, fibrosis, and lipidosis ${ }^{4,9}$. These muscle disorders present an exceptional challenge to producers, as dietary or management strategies against them often fail to improve meat quality ${ }^{10,11}$ or lack viability due to impaired live performance ${ }^{12}$ or cost-prohibitive inputs. The tight association between breast muscle disorders and economic traits such as feed efficiency and breast muscle yield suggests that successful mitigation of meat quality defects without simultaneous compromise to desirable traits will require an understanding of the genetic basis of these myopathies and selection against their causal variants.

Several hypotheses exist regarding the underlying causes of wooden breast and white striping. Some implicate the rapid growth of the pectoralis major and relative vascular deficiency for creating a buildup of waste products and hypoxic conditions ${ }^{13,14}$ in the breast muscle, while others suggest shared etiologic underpinnings with type

${ }^{1}$ Center for Bioinformatics and Computational Biology, University of Delaware, Newark, DE, USA. ${ }^{2}$ Department of Animal and Food Sciences, University of Delaware, Newark, DE, USA. ${ }^{3}$ Department of Animal Science, lowa State University, Ames, IA, USA. ${ }^{\boxplus}$ email: abasht@udel.edu 


\begin{tabular}{|l|l|l|l|l|c|}
\hline Trait & Number of samples & Mean & SD & Heritability & Residual variance \\
\hline Wooden Breast & 1193 & 1.57 & 1.06 & $0.49 \pm 0.06$ & $0.51 \pm 0.05$ \\
\hline White Striping & 1193 & 1.14 & 0.77 & $0.50 \pm 0.06$ & $0.28 \pm 0.03$ \\
\hline Body Weight 13d $(\mathrm{g})$ & 1193 & 354 & 57 & $0.36 \pm 0.06$ & $1,548 \pm 124$ \\
\hline Body Weight 7wk (g) & 1193 & 3496 & 449 & $0.40 \pm 0.06$ & $45,085 \pm 3741$ \\
\hline
\end{tabular}

Table 1. Trait statistics and estimates $( \pm$ SE) of heritability and residual variance from univariate analyses of wooden breast, white striping, and body weight at 13 days and at 7 weeks of age. Wooden breast was scored on a 5 -point scale from 0 -Normal to 4 -Severe. White striping was scored on a 4 -point scale from 0 -Normal to 3-Severe.

\begin{tabular}{|l|c|l|l|l|}
\hline & Wooden breast & White striping & Body weight 13d & Body weight 7wk \\
\hline Wooden breast & & $0.65 \pm 0.02$ & $0.12 \pm 0.03$ & $0.25 \pm 0.03$ \\
\hline White striping & $0.88 \pm 0.04$ & & $0.17 \pm 0.03$ & $0.19 \pm 0.03$ \\
\hline Body weight 13d & $-0.04 \pm 0.12$ & $0.15 \pm 0.12$ & & $0.49 \pm 0.03$ \\
\hline Body weight 7wk & $0.16 \pm 0.11$ & $0.09 \pm 0.11$ & $0.54 \pm 0.09$ & \\
\hline
\end{tabular}

Table 2. Estimates ( \pm SE) of phenotypic (above diagonal) and genetic (below diagonal) correlations among wooden breast, white striping, and body weight at 13 days and at 7 weeks of age based on bivariate analyses.

2 diabetes and other metabolic disorders in mammals ${ }^{15,16}$. However, current knowledge regarding the genetic basis of wooden breast and white striping is extremely limited and somewhat conflicting. Bailey et al. ${ }^{17}$ estimated heritability $\left(\mathrm{h}^{2}\right)$ of wooden breast and white striping in a purebred commercial broiler line to be low -0.07 for wooden breast and 0.25 for white striping-but also demonstrated a dramatic $18.4 \%$ reduction in wooden breast incidence after only 2 years of genetic selection against breast muscle myopathies. Another study of two broiler lines divergently selected for ultimate $\mathrm{pH}$ of the pectoralis major estimated heritability $\left(\mathrm{h}^{2}\right)$ of white striping to be $0.65^{18}$. The only genome-wide association study of white striping was performed on a similar population and found no markers with genome-wide significance ${ }^{19}$, while the genetic architecture of wooden breast currently remains unexplored.

Our poor understanding of these traits at the genetic level precludes our ability to adequately mitigate their effects through either broiler breeding or management. Therefore, the aim of this study is to estimate genetic parameters for wooden breast, white striping, and two body weight traits in a hybrid commercial broiler population and to identify quantitative trait loci (QTLs) and candidate genes to elucidate potential molecular mechanisms contributing to myopathy development.

\section{Results and discussion}

Trait statistics and genetic parameter estimates. Of the 1,194 progeny that were genotyped for this study, only one bird did not meet sequence filter criteria and was excluded from all analyses. Sex chromosomes were used to confirm each bird's sex, and found that 12 birds were mis-gendered at necropsy. The sex-specific and overall distributions of wooden breast and white striping scores for the 1,193 birds that passed filter criteria can be found in Supplementary Table S1. The prevalence of wooden breast, i.e. the proportion of birds with a wooden breast score greater than 0 , was approximately $79 \%$, although the scoring system implemented in this study was relatively sensitive to mild signs of the myopathy compared to other studies ${ }^{10,12}$. Meat quality is not substantially affected in birds with scores of 1 or 2, and severe wooden breast (score of 4) was detected only in $2.1 \%$ of chickens in our study (Supplementary Table S1). The prevalence of white striping was similarly high, at approximately $80 \%$. Compared to males, female birds exhibited a lower prevalence of wooden breast $(71 \%$ vs. $87 \%$; $p$-value $<0.001)$ and white striping $(73 \%$ vs. $87 \%$; $p$-value $<0.001)$.

Descriptive statistics, heritability estimates, and variance components of the traits are summarized in Table 1. In our population, the wooden breast phenotype exhibited substantially higher heritability $\left(h^{2}=0.49\right)$ than indicated by previous work, which estimated heritability between 0.1 and $0.24^{20}$. Additionally, we found the heritability of white striping $\left(h^{2}=0.50\right)$ to lie between previous estimates ranging from $0.18^{20}$ to $0.65^{18}$. Variability among these studies is not surprising, as heritability is inherently specific to the population and environment in which it is estimated. For example, the lower estimates for heritability of wooden breast and white striping were for purebred commercial broiler lines that had relatively low incidence rates of myopathy- $0.16 \%$ to $0.39 \%$ for wooden breast; $14.46 \%$ to $49.6 \%$ for white striping-compared to other hybrid commercial broiler populations ${ }^{20}$. Moderate heritability for wooden breast and white striping indicates that both genetic and environmental factors exert strong influences on phenotypic differences for these traits, which is consistent with studies that have demonstrated the ability to reduce severity and incidence of breast muscle myopathies through both genetic selection ${ }^{17}$ and manipulation of dietary energy ${ }^{12,21}$.

Our analysis found genetic correlations (Table 2) of wooden breast and white striping with body weight at 13 days ( -0.04 and 0.15 respectively) and body weight at 7 weeks ( 0.16 and 0.09 respectively) to be low, while the estimate of the genetic correlation between the two myopathies was high (0.88). The latter reinforces an existing 

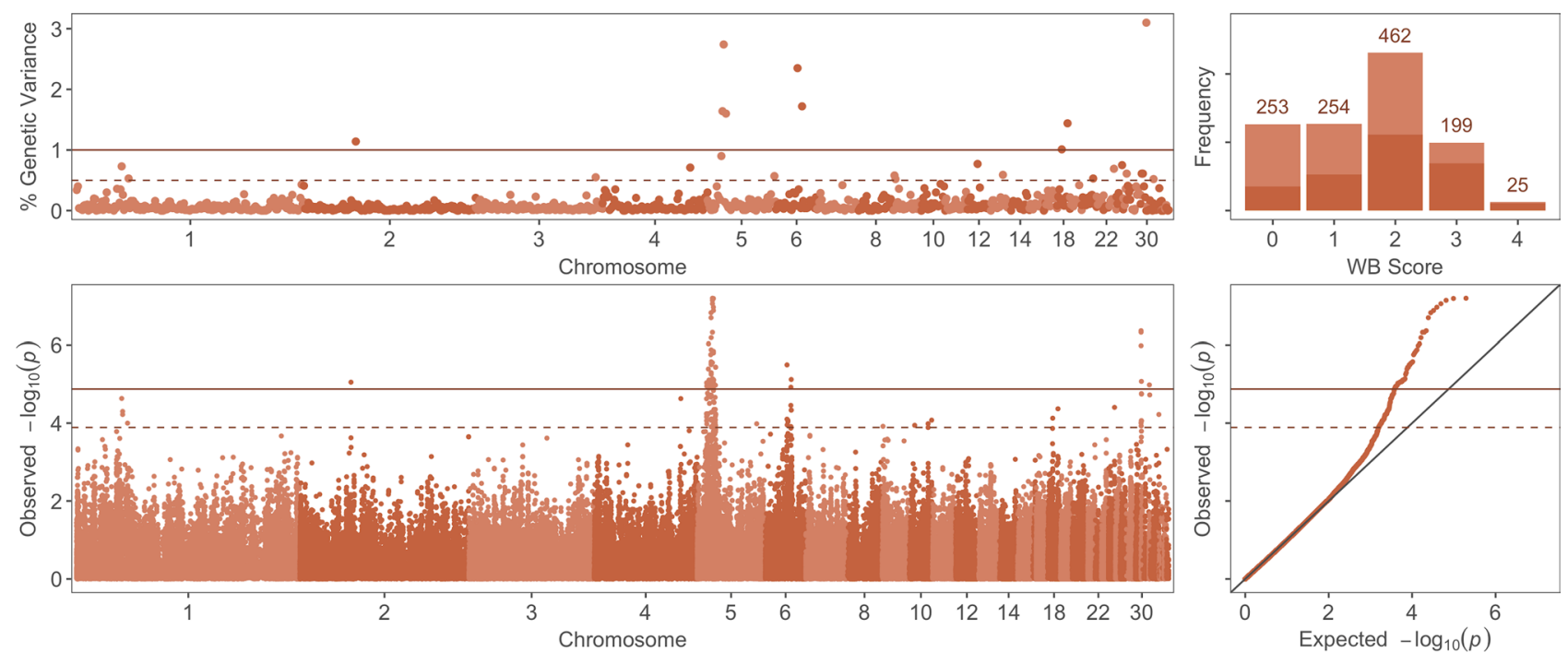

Figure 1. Genome-wide association results for wooden breast (WB) score using multi-marker (BayesB) and single-SNP (mixed linear model) analyses. (Top Left) Percentage of genetic variance explained by $1-\mathrm{Mb}$ regions across the genome for WB score. (Top Right) Distribution of WB scores across progeny used in genome-wide association analyses; dark red $=$ male, light red $=$ female. (Bottom Left) Manhattan plot of single-SNP results showing the $-\log 10$ ( $p$-value) of SNPs ordered by chromosome and position. (Bottom Right) Quantile-quantile plot of $p$-values from single-SNP results of WB score. Solid and dashed lines indicate significant and suggestive thresholds, respectively, for each model.

hypothesis that these two traits are related to each other and may be variations of the same disorder ${ }^{7,8}$. Note that low genetic correlation between the muscle disorders and body weight traits does not signify low genetic correlations of muscle disorders with other performance traits that were not assessed in this study, such as breast muscle yield and feed conversion ratio.

Sequence and SNP statistics. Excluding the single sample that did not meet filter criteria, sequencing produced an average of 5.88 million reads per sample, with an average mapping rate of $77.3 \%$. A total of 199,957 SNPs were retained after locus filtering, consisting of 195,617 autosomal SNPs and 4340 SNPs on the Z chromosome. The average sequence depth for each genotyped SNP was 10.8, with $97.9 \%$ of all genotype calls supported by at least $1 \mathrm{read}$. The average minor allele frequency was 0.24 . Filter criteria implemented in this study were substantially more stringent than in previous studies utilizing low-depth genotyping-by-sequencing methods ${ }^{22}$ in order to identify QTLs with greater confidence.

Linkage disequilibrium. Linkage disequilibrium $\left(\mathrm{r}^{2}\right)$ in the population decayed at an extremely rapid rate (Supplementary Table S2), dropping below 0.2 at a distance of 2092 base pairs (bp) on macro-chromosomes, at $1410 \mathrm{bp}$ on intermediate chromosomes, and at $1046 \mathrm{bp}$ on micro-chromosomes. This value was substantially higher on the $\mathrm{Z}$ chromosome, at about $78 \mathrm{~kb}$, because this chromosome is subjected to lower recombination frequency and a smaller effective population size. Based on $\mathrm{D}_{0.2}$ estimates for each chromosome group, SNPs in the current dataset encompassed $27.5 \%$ of the genome (autosomes and $\mathrm{Z}$ chromosome), with chromosome-specific capture rates ranging from $84.6 \%$ on the $\mathrm{Z}$ chromosome to $3.33 \%$ on chromosome 31 (Supplementary Table S3).

GWAS results for wooden breast. Single-SNP analysis of the genetic basis of wooden breast identified 51 SNPs that were significantly associated and an additional 71 SNPs that were suggestively associated with wooden breast score (Fig. 1; Supplementary Table S4). These loci are located across 11 chromosomes of the Gallus gallus genome, including (GGA) 1,2, 4, 5, 6, 9, 10, 18, 24, 30, and 32; however, the majority of significant markers (42 SNPs, 82\%) and significant/suggestive markers combined (84 SNPs, 69\%) were confined to a region on GGA5 from 8.1 to $16.4 \mathrm{Mb}$. A total of 28 QTLs on chromosomes (GGA) 2, 5, 6, 30, and 32 were identified from significant SNPs that were clustered based on LD (Table 3). Bayesian multi-marker regression corroborated results from single-SNP analysis, detecting 91 -Mb windows, on chromosomes (GGA) 2, 5, 6, 18, and 30, that each explained more than $1 \%$ of genetic variance for the trait (Table 4). Together, these 9 windows explained $16.7 \%$ of the genetic variance for wooden breast. Three windows on GGA5, from 13.0 to $17.0 \mathrm{Mb}$, explained a total of $6 \%$ of genetic variance, although the single highest-ranking window in this analysis was at the beginning of GGA30, which explained 3.1\% of genetic variance. Although no significant QTLs were identified for sex-specific analyses of wooden breast, some markers exceeded the suggestive threshold in female birds and are listed in Supplementary Table S5. Genomic windows with suggestive significance in the BayesB analyses are listed in Supplementary Table S6 for all traits.

Top candidate genes for wooden breast (Table 3) include cyclin dependent kinase inhibitor $1 C$ (CDKN1C), cathepsin D (CTSD), potassium voltage-gated channel subfamily Q member 1 (KCNQ1), lymphocyte-specific protein 1 


\begin{tabular}{|c|c|c|c|c|c|c|c|c|c|c|}
\hline \multicolumn{4}{|l|}{ QTL region } & \multicolumn{6}{|c|}{ Top SNP in QTL region } & \multirow{2}{*}{$\begin{array}{l}\text { "1"> } \\
\text { Candidate genes }\end{array}$} \\
\hline Chromosome & Start (bp) & End (bp) & $\begin{array}{l}\text { Number of } \\
\text { significant } \\
\text { SNPs }\end{array}$ & Position (bp) & $\begin{array}{l}\text { Alleles (Effect/ } \\
\text { Alternative) }\end{array}$ & $\begin{array}{l}\text { Effect allele } \\
\text { frequency }\end{array}$ & $\begin{array}{l}\text { Effect size } \\
\text { estimate }\end{array}$ & p-value & FDR & \\
\hline 2 & - & - & 1 & 45199243 & $\mathrm{~T} / \mathrm{C}$ & 0.473 & 0.211 & $8.91 \mathrm{e}-06$ & 0.043 & - \\
\hline 5 & 8327574 & 8393723 & 3 & 8393723 & $\mathrm{~A} / \mathrm{T}$ & 0.228 & 0.289 & $9.17 \mathrm{e}-06$ & 0.043 & \begin{tabular}{|l|} 
MICAL2 \\
DKK3
\end{tabular} \\
\hline 5 & - & - & 1 & 9797353 & $\mathrm{~A} / \mathrm{G}$ & 0.240 & 0.305 & $4.10 \mathrm{e}-06$ & 0.031 & NRIP3 \\
\hline 5 & 9975375 & 10239083 & 2 & 9975375 & $\mathrm{~T} / \mathrm{C}$ & 0.232 & 0.325 & $9.28 \mathrm{e}-07$ & 0.014 & $\begin{array}{l}\text { DENND2B } \\
\text { RPL27A }\end{array}$ \\
\hline 5 & - & - & 1 & 10415181 & T/A & 0.390 & 0.246 & $7.83 e-06$ & 0.043 & - \\
\hline 5 & - & - & 1 & 10675242 & T/A & 0.181 & 0.291 & $1.10 \mathrm{e}-05$ & 0.046 & PSMA1 \\
\hline 5 & 11903835 & 11903902 & 4 & 11903836 & G/A & 0.235 & -0.197 & $8.68 \mathrm{e}-06$ & 0.043 & - \\
\hline 5 & - & - & 1 & 11913861 & $\mathrm{~A} / \mathrm{G}$ & 0.163 & -0.305 & $1.15 \mathrm{e}-05$ & 0.046 & - \\
\hline 5 & - & - & 1 & 11924135 & $\mathrm{~T} / \mathrm{C}$ & 0.255 & 0.300 & $1.76 \mathrm{e}-06$ & 0.019 & - \\
\hline 5 & 12196653 & 12196751 & 2 & 12196653 & $\mathrm{~T} / \mathrm{C}$ & 0.232 & -0.352 & $1.50 \mathrm{e}-07$ & 0.004 & USH1C \\
\hline 5 & 12310175 & 12310343 & 7 & 12310234 & $\mathrm{~T} / \mathrm{C}$ & 0.244 & -0.270 & $6.52 \mathrm{e}-07$ & 0.011 & - \\
\hline 5 & 13374229 & 13429787 & 2 & 13429787 & $\mathrm{~T} / \mathrm{C}$ & 0.240 & -0.313 & $4.75 \mathrm{e}-07$ & 0.008 & \begin{tabular}{|l|} 
KCNQ1 \\
SLC22A18 \\
CDKN1C \\
\end{tabular} \\
\hline 5 & 13449328 & 13449379 & 3 & 13449367 & $\mathrm{~T} / \mathrm{C}$ & 0.369 & -0.281 & $6.38 \mathrm{e}-08$ & 0.004 & KCNQ1 \\
\hline 5 & - & - & 1 & 13508017 & $\mathrm{C} / \mathrm{A}$ & 0.245 & 0.301 & $7.27 \mathrm{e}-06$ & 0.043 & KCNQ1 \\
\hline 5 & - & - & 1 & 13899194 & $\mathrm{G} / \mathrm{C}$ & 0.255 & -0.284 & $1.15 \mathrm{e}-05$ & 0.046 & - \\
\hline 5 & 14280580 & 14280613 & 2 & 14280613 & $\mathrm{~T} / \mathrm{C}$ & 0.335 & 0.311 & $6.47 \mathrm{e}-08$ & 0.004 & LSP1 \\
\hline 5 & 14295515 & 14308280 & 4 & 14301905 & $\mathrm{G} / \mathrm{C}$ & 0.180 & -0.307 & $1.44 \mathrm{e}-06$ & 0.018 & \begin{tabular}{|l|} 
TNNI2 \\
SYT8 \\
ENSGALG00000006608
\end{tabular} \\
\hline 5 & 14321077 & 14321102 & 2 & 14321077 & G/A & 0.433 & 0.280 & $1.32 \mathrm{e}-07$ & 0.004 & \begin{tabular}{|l|} 
CTSD \\
ENSGALG00000006608
\end{tabular} \\
\hline 5 & - & - & 1 & 14529930 & $\mathrm{~T} / \mathrm{C}$ & 0.286 & 0.065 & $7.87 e-06$ & 0.043 & \begin{tabular}{|l|} 
BRSK1 \\
ENSGALG00000006608
\end{tabular} \\
\hline 5 & - & - & 1 & 16063707 & $\mathrm{~T} / \mathrm{C}$ & 0.174 & 0.319 & $1.06 \mathrm{e}-05$ & 0.046 & $\begin{array}{l}\text { ENSGALG00000044313 } \\
\text { PHRF1 }\end{array}$ \\
\hline 5 & 16219365 & 16219383 & 2 & 16219365 & $\mathrm{~A} / \mathrm{G}$ & 0.213 & -0.292 & $3.66 \mathrm{e}-06$ & 0.029 & HRAS \\
\hline 6 & - & - & 1 & 19640631 & $\mathrm{~T} / \mathrm{G}$ & 0.389 & 0.343 & $3.21 \mathrm{e}-06$ & 0.027 & - \\
\hline 6 & - & - & 1 & 23100280 & $\mathrm{~A} / \mathrm{G}$ & 0.303 & 0.294 & $1.20 \mathrm{e}-05$ & 0.047 & $\begin{array}{l}\text { GOT1 } \\
\text { CNNM1 }\end{array}$ \\
\hline 6 & 23389752 & 23391597 & 3 & 23389752 & $\mathrm{~A} / \mathrm{G}$ & 0.119 & 0.368 & $7.59 \mathrm{e}-06$ & 0.043 & $L C O R$ \\
\hline 30 & - & - & 1 & 90012 & $\mathrm{C} / \mathrm{T}$ & 0.292 & -0.249 & $4.34 \mathrm{e}-07$ & 0.008 & $\begin{array}{l}\text { DNM2 } \\
\text { QTRT1 }\end{array}$ \\
\hline 30 & 96694 & 97362 & 2 & 97362 & $\mathrm{~A} / \mathrm{G}$ & 0.196 & -0.283 & $4.73 e-07$ & 0.008 & DNM2 \\
\hline 30 & - & - & 1 & 393145 & $\mathrm{~A} / \mathrm{G}$ & 0.275 & 0.240 & $8.50 \mathrm{e}-06$ & 0.043 & \begin{tabular}{|l|} 
CNN1 \\
ZNF653 \\
ECSIT \\
ELOF1 \\
ACP5
\end{tabular} \\
\hline 32 & - & - & 1 & 86047 & $\mathrm{C} / \mathrm{G}$ & 0.297 & -0.261 & $1.05 \mathrm{e}-05$ & 0.046 & $\begin{array}{l}\text { ACTN4 } \\
\text { ECH1 }\end{array}$ \\
\hline
\end{tabular}

Table 3. Wooden Breast QTL regions containing SNPs with FDR adjusted $p$-values less than 0.05 . Results of single-marker genome-wide association analysis using all birds. Candidate genes include protein-coding genes located within $5000 \mathrm{bp}$ upstream or downstream of the QTL start or end site, respectively.

(LSP1), solute carrier family 22 member 18 (SLC22A18) and USH1 protein network component harmonin (USH1C) on GGA5 and dynamin 2 (DNM2) on GGA30. In humans, variation in all of these genes has been linked to altered insulin expression or secretion ${ }^{23-26}$ despite their having distinct biological functions.

GWAS results for white striping. A total of 18 SNPs (10 significant and 8 suggestive) were associated with white striping score (Fig. 2; Supplementary Table S7), all of which are located on GGA5, except for one marker on GGA11. Similar to the wooden breast association analysis results, most of these loci (9 significant and 6 suggestive, $83 \%$ of combined) were on GGA5, between 12.1 and $14.8 \mathrm{Mb}$ (Table 5). Bayesian multi-marker regression identified a window in this same region, between 14.0 and $15.0 \mathrm{Mb}$, as the highest-ranking $1-\mathrm{Mb}$ window, explaining $5.4 \%$ of genetic variance (Table 4 ). Two other windows also explain more than $1 \%$ of genetic variance, one on the long arm of GGA5 and one on GGA12. Together, the three significant windows for white striping explained $9.8 \%$ of the genetic variance for the trait. None of the QTLs identified in this study overlapped 


\begin{tabular}{|l|l|l|l|l|l|}
\hline Trait & Chromosome & Window $(\mathbf{M b})$ & \# SNPs & Explained genetic variance (\%) & $\boldsymbol{p}>\mathbf{0}^{\mathbf{1}}$ \\
\hline \multirow{5}{*}{ Wooden Breast } & 30 & $0-1$ & 327 & 3.10 & 0.970 \\
\cline { 2 - 6 } & 5 & $14-15$ & 186 & 2.74 & 0.808 \\
\cline { 2 - 6 } & 6 & $19-20$ & 82 & 2.35 & 0.775 \\
\cline { 2 - 6 } & 6 & $23-24$ & 990 & 1.72 & 0.951 \\
\cline { 2 - 6 } & 5 & $13-14$ & 224 & 1.64 & 0.622 \\
\cline { 2 - 6 } & 5 & $16-17$ & 436 & 1.60 & 0.798 \\
\cline { 2 - 6 } & 18 & $9-10$ & 1508 & 1.44 & 0.987 \\
\cline { 2 - 6 } & 2 & $45-46$ & 147 & 1.14 & 0.587 \\
\hline \multirow{5}{*}{ White Striping } & 5 & $4-5$ & 791 & 1.01 & 0.990 \\
\cline { 2 - 6 } & 5 & $14-15$ & 186 & 5.38 & 0.990 \\
\cline { 2 - 6 } & 12 & $58-59$ & 604 & 3.18 & \\
\hline
\end{tabular}

Table 4. Results of Bayesian multi-marker regression for wooden breast and white striping. Windows identified by Bayes $B$ were considered significant if they explained $\geq 1 \%$ of genetic variance. Body weight traits did not have any significant $1-\mathrm{Mb}$ windows. $\mathrm{P}>0$ refers to the frequency of sample for which the region had a nonzero effect.
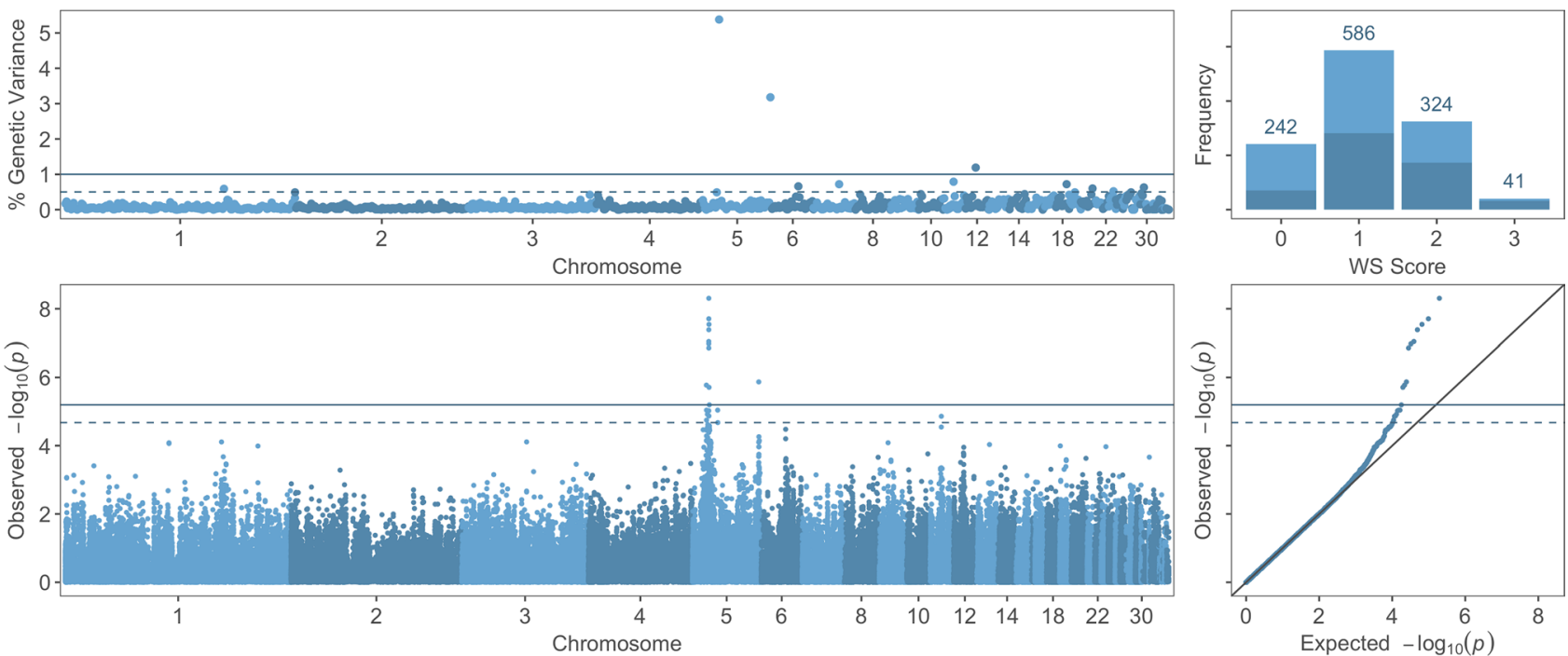

Figure 2. Genome-wide association results for white striping (WS) score using multi-marker (BayesB) and single-SNP (mixed linear model) analyses. (Top Left) Percentage of genetic variance explained by 1-Mb regions across the genome for WS score. (Top Right) Distribution of WS scores across progeny used in genome-wide association analyses; dark blue $=$ male, light blue $=$ female. $($ Bottom Left $)$ Manhattan plot of single-SNP results showing the $-\log 10$ ( $p$-value) of SNPs ordered by chromosome and position. (Bottom Right) Quantile-quantile plot of p-values from single-SNP results of WS score. Solid and dashed lines indicate significant and suggestive thresholds, respectively, for each model.

with QTLs previously identified for white striping; Pampouille et al. ${ }^{19}$ did not discover QTLs with genome-wide significance, but identified three QTLs with chromosome-wide significance in the 3rd Mb windows of GGA17 and GGA18 and in the 88th Mb window of GGA1. No significant or suggestive SNPs were identified for sexspecific analyses of white striping. Genomic windows with suggestive significance in the BayesB analyses are listed in Supplementary Table S6.

Top candidate genes for white striping include CTSD, LSP1, troponin I2 fast skeletal type (TNNI2), synaptotagmin 8 (SYT8), and MOB kinase activator 2 (MOB2). As with candidate genes for wooden breast, variation in mammalian homologues of all of these genes is linked to altered insulin expression or secretion in pancreatic beta cells $s^{25,27}$. However, they possess additional functions that may be relevant to poultry myopathies, such as calcium-dependent regulation of striated muscle contraction ${ }^{28}$ (TNNI2), and regulation neutrophil transendothelial migration ${ }^{29}$ (LSP1).

GWAS results for body weight traits. No QTLs were identified for either body weight trait using singleSNP or multi-marker analyses (Supplementary Figs. S1 and S2; Supplementary Table S6). This may be a result 


\begin{tabular}{|c|c|c|c|c|c|c|c|c|c|c|}
\hline \multicolumn{4}{|l|}{ QTL } & \multicolumn{7}{|c|}{ Top SNP in QTL } \\
\hline Chromosome & Start (bp) & End (bp) & $\begin{array}{l}\text { Number of } \\
\text { significant } \\
\text { SNPs }\end{array}$ & Position (bp) & $\begin{array}{l}\text { Alleles (Effect/ } \\
\text { Alternative) }\end{array}$ & $\begin{array}{l}\text { Effect allele } \\
\text { frequency }\end{array}$ & $\begin{array}{l}\text { Effect size } \\
\text { estimate }\end{array}$ & $p$-value & FDR & Candidate genes \\
\hline 5 & - & - & 1 & 12196653 & $\mathrm{~T} / \mathrm{C}$ & 0.232 & -0.239 & $1.68 \mathrm{e}-06$ & 0.036 & USH1C \\
\hline 5 & 14280580 & 14320311 & 4 & 14280613 & $\mathrm{~T} / \mathrm{C}$ & 0.335 & 0.228 & $9.07 \mathrm{e}-08$ & 0.003 & $\begin{array}{l}\text { LSP1 } \\
\text { TNNI2 } \\
\text { SYT8 } \\
\text { ENSGALG00000006608 } \\
\text { CTSD }\end{array}$ \\
\hline 5 & 14321077 & 14321102 & 2 & 14321077 & G/A & 0.433 & 0.230 & $4.93 \mathrm{e}-09$ & 0.001 & $\begin{array}{l}\text { ENSGALG00000006608 } \\
\text { CTSD }\end{array}$ \\
\hline 5 & - & - & 1 & 14477401 & $\mathrm{~T} / \mathrm{C}$ & 0.238 & -0.241 & $2.86 \mathrm{e}-08$ & 0.002 & $\begin{array}{l}\text { ENSGALG00000006608 } \\
\text { MOB2 }\end{array}$ \\
\hline 5 & - & - & 1 & 14529930 & $\mathrm{~T} / \mathrm{C}$ & 0.286 & 0.229 & $1.95 \mathrm{e}-06$ & 0.038 & $\begin{array}{l}\text { ENSGALG00000006608 } \\
\text { BRSK1 }\end{array}$ \\
\hline 5 & - & - & 1 & 58074636 & $\mathrm{~A} / \mathrm{G}$ & 0.359 & 0.166 & $1.35 \mathrm{e}-06$ & 0.033 & NIN \\
\hline
\end{tabular}

Table 5. White Striping QTL. Results of single-marker genome-wide association analysis using all progeny. Candidate genes include protein-coding genes located within $5000 \mathrm{bp}$ upstream or downstream of the QTL start or end site, respectively.

extreme selection for growth rate over many decades, likely resulting in fixed alleles at loci of large effect. By contrast, wooden breast and white striping have a much shorter selection history based on their somewhat recent appearance, and have potentially been subjected to both positive and negative selection if they indeed share a strong genetic basis with performance traits such as feed efficiency and breast muscle yield. This would preserve variation at loci with moderate or large effects, which are easier to detect with lower sample sizes in genome-wide association analyses.

QTL detection for both body weight and muscle disorder traits may also have been hindered by the extremely rapid LD decay (Supplementary Table S2) in our 4-way crossbred broiler population relative to the genome coverage achieved by our genotyping methods and filter criteria. Using SNP positions and $\mathrm{D}_{0.2}$, we estimated that only $27.52 \%$ of autosomes and the $\mathrm{Z}$ chromosome were encompassed by SNPs in this study. This is a conservative estimate, as it includes chromosomal regions with extremely low variation, but indicates the strong potential for additional QTLs with large effects that were not captured by this study. Our sample size also prevented detection of small-effect QTLs by limiting statistical power. The rapid LD decay, however, gives us confidence that the QTLs detected in this study are in close proximity to their causal mutations.

Comparative genomics of GGA5. Chicken chromosome 5 (GGA5) has conserved synteny with portions of human chromosomes 11, 14, and $15^{30}$. The majority of QTLs identified for both wooden breast and white striping were located in a region that has highly conserved synteny with HSA11 (Fig. 4). Indeed, there is greater conservation of synteny for genes on HSA11 between chickens and humans than between mice and humans, although numerous intrachromosomal rearrangements are present. The area on HSA11 that is homologous to QTL regions on GGA5 identified in the present study is associated with numerous metabolic and growth disorders and various forms of diabetes mellitus (Fig. 4). For example, KCNQ1 locus in this region is heavily studied in humans as part of an imprinted gene cluster that when dysregulated can cause overgrowth disorders including Beckwith-Wiedemann syndrome ${ }^{31}$. Even though the size of the region and order of genes is nearly identical between chickens and mammals (Fig. 4), chickens lack the relevant imprinting control elements present in mammals ${ }^{32}$. This suggests that chickens may serve as an excellent model for studying topics such as evolutionary genomics, imprinting control, and growth disorders.

The QTL-rich region of GGA5 also contains several areas that show evidence of strong selection sweeps (Fig. 4) in two commercial purebred male broiler lines selected for breast muscle growth and feed efficiency ${ }^{33}$. Selective breeding for meat production traits is considered a major culprit for the rapid increase in meat quality issues among broiler chickens, and the genomic co-localization of loci that are beneficial for production traits with loci associated with breast muscle myopathies provides further evidence of this. Targeted sequencing of GGA5 is required to determine if the specific genetic mutations contributing to each trait are simply linked or, in fact, the same. Additionally, targeted sequencing of GGA5 with long-read technologies could reveal copy number variants, which are not captured by SNP data. The SRY-box transcription factor 6 (SOX6) gene is of particular interest because it was identified as a candidate gene for the selection sweep region on GGA $5^{33}$. Although SOX6 was not identified as a candidate gene for wooden breast based on association analyses described here, a selection sweep in or near this gene would hinder detection of causal variants using many GWAS methods due to filters for minor allele frequency.

Clinical significance of candidate genes. A major functional theme of candidate genes identified in this study is insulin secretion and action, particularly in the QTL-rich region of GGA5 (Fig. 3), which contains numerous genes previously shown to be associated with metabolic and cardiac disorders in humans (Fig. 4). For example, KCNQ1 polymorphisms are associated with altered insulin secretion in pancreatic $\beta$ cells, short and 


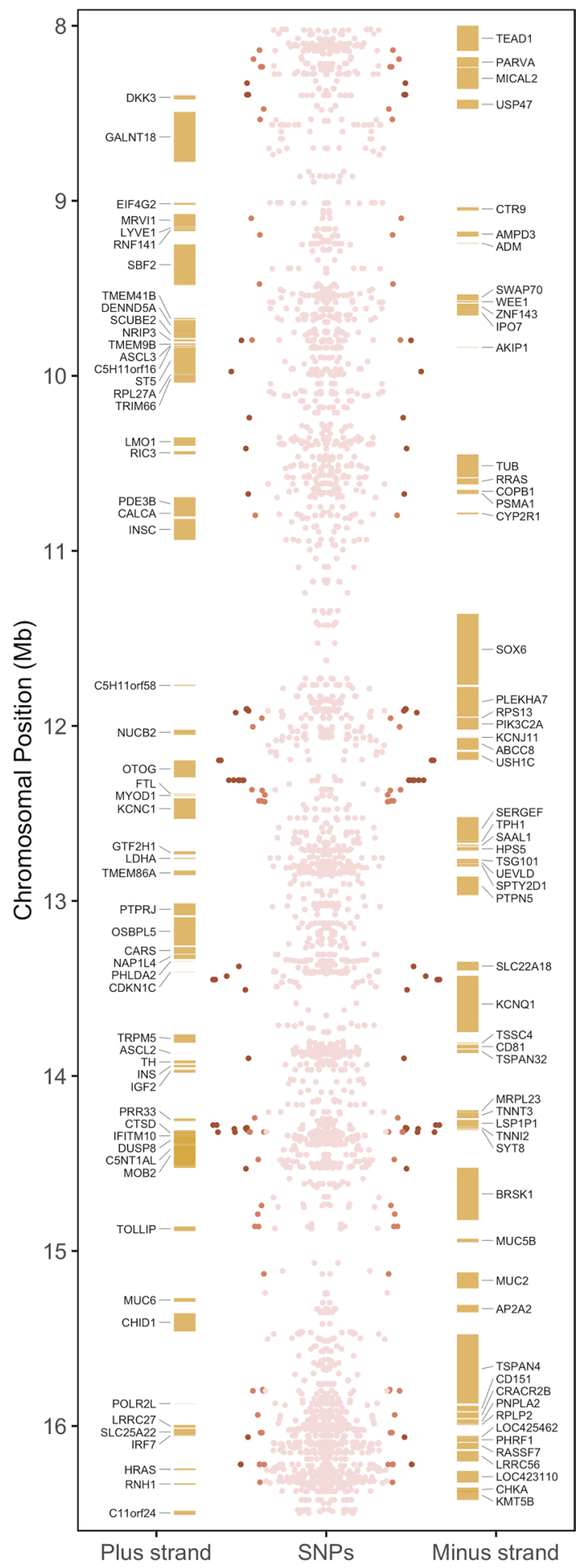

Figure 3. Gene landscape of QTL-rich region between $8.0 \mathrm{Mb}$ and $16.5 \mathrm{Mb}$ on chicken chromosome 5 . Mirrored Manhattan plot shows SNPs with significance thresholds indicated by color; dark red = significant, orange $=$ suggestive .

long QT syndrome in cardiac muscle, and sensorineural deafness in the inner ear ${ }^{34}$. This gene encodes a highlystudied voltage-gated potassium channel, which interacts with numerous other protein subunits and switches from voltage-dependent to constitutive activity ${ }^{34}$, endowing it with a diverse set of functional roles and associating it with a proportionally diverse number of pathologies. Some pleiotropic KCNQ1 variants in humans can simultaneously increase insulin secretion in the pancreas, reduce serum potassium upon oral glucose challenge, and cause long QT syndrome, putting individuals at risk of sudden, uncontrollable, arrhythmias which may lead to fainting or sudden death ${ }^{35}$. This could represent a potential cause of sudden cardiac death reported in 


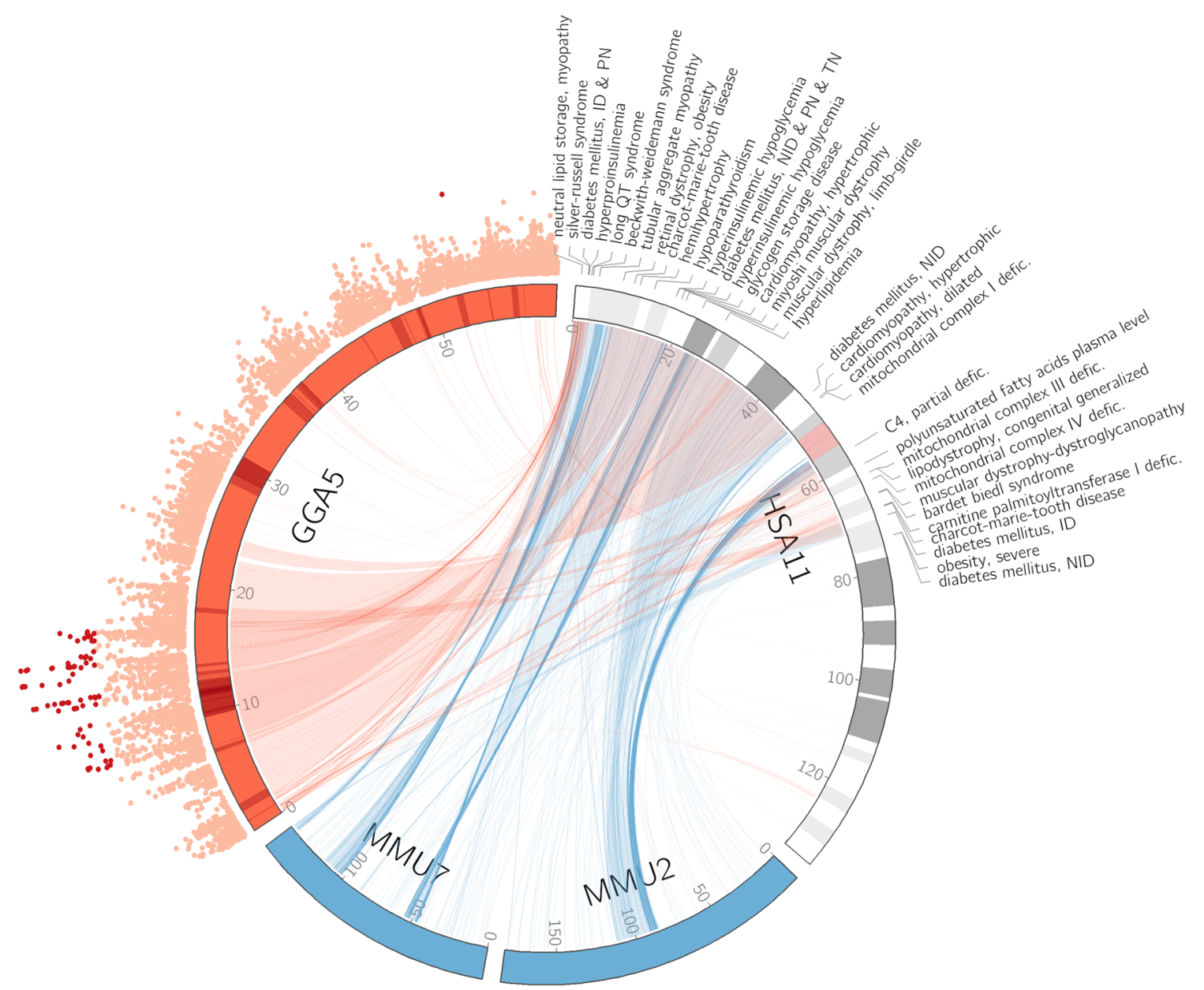

Figure 4. Conserved synteny between chickens and humans. The wooden breast QTL-rich region of chicken chromosome 5 (GGA5) has highly conserved synteny with human chromosome 11 (HSA11), especially compared to homologous regions in the mouse genome on chromosomes 2 (MMU2) and 7 (MMU7). In humans, this area is associated with a high number of growth and metabolic disorders, which are highlighted here based on existing knowledge of wooden breast and white striping, including a hypothesis suggesting dysregulation of lipid and glucose metabolism as an important underlying factor in development of the myopathies. The scatter plot on GGA5 represents GWAS results from the present study with significant SNPs in red. Red bands on GGA5 indicate previously identified selective sweeps in commercial purebred broiler lines that are susceptible to wooden breast with overlapping selective sweep regions in dark red. The centromere (pink) and G-banding (grey and white) are shown on HSA11 to improve visualization. Defic: deficiency, ID: insulin-dependent, NID: non-insulin-dependent, PN: permanent neonatal, TN: transient neonatal.

broilers, especially those with wooden breast ${ }^{36}$. We re-examined data from a previous study of differential gene expression associated with wooden breast in 2 -week-old female broilers ${ }^{16}$ and found that, although there was no differential expression of KCNQ1 in the pectoralis major of 2-week-old birds, expression levels of the gene were very low in all birds (data not shown). It is therefore likely that genetic variation in KCNQ1 would primarily affect organs other than the pectoralis major, potentially including the pancreas.

Some candidate genes on GGA5 affect insulin secretion through both gene-gene interactions and protein function, as the insulin gene (INS) is located in the middle of this QTL-rich region at approximately $13.94 \mathrm{Mb}$ (Fig. 3). For instance, synaptotagmin 8 (SYT8) encodes a membrane protein with roles in trafficking and exocytosis, including insulin secretion in pancreatic islet cells ${ }^{25}$. The SYT8 gene is located over $300 \mathrm{~kb}$ away from INS in humans, and yet physically interacts with the INS locus to elevate SYT8 expression, especially in the presence of glucose $\mathrm{e}^{25}$. This indicates the importance of SYT8 for both basal and glucose-stimulated insulin secretion in human islets. The same study identified additional interactions of INS with troponin I2, fast skeletal type (TNNI2) and lymphocyte-specific protein 1 (LSP1), which are also top candidate genes for wooden breast and white striping.

QTLs outside of GGA5 have strong ties to insulin as well. The protein encoded by DNM2 on GGA30 is critical for proper pancreatic function, where it regulates biphasic insulin secretion and glucose homeostasis in mammals. Knockout of DNM2 in pancreatic $\beta$ cells in mice causes glucose intolerance via remodeling of the actin cytoskeleton and inefficient endocytosis-exocytosis coupling, indicating a potential pathophysiological link between DNM2 function and diabetes mellitus ${ }^{24}$. DNM2 is best known for its involvement in two distinct congenital neuromuscular diseases of humans, centronuclear myopathy and Charcot-Marie-Tooth neuropathy. 
Several of the characteristic features of DNM2-associated centronuclear myopathy are reminiscent of the histopathological signs of wooden breast and white striping in broiler breast muscle, including increased variability of myofiber size, endomysial fibrosis, calcium homeostasis alterations, and abnormal centralization of nuclei in muscle fibers, which can also be seen in regenerating myofibers ${ }^{37,38}$. Although not discussed by Mutryn et al. ${ }^{8}$, DNM2 expression was found to be downregulated in wooden breast-affected broilers compared to unaffected broilers at market age (data not shown), which is of particular interest considering findings of a knockout study of this gene in mouse gastrocnemius muscle. Tinelli et al. ${ }^{39}$ found that DNM2 knockout caused a decrease in the number of muscle fibers, an increase in the proportion of smaller muscle fibers, an increase in lipid droplets, mitochondrial enlargement and disruption of cristae, mitochondrial dysfunction, and altered neuromuscular junctions at various developmental ages ${ }^{39}$. Previously, Papah et al. ${ }^{5}$ described an increase in acellular lipid droplets and abnormal mitochondrial morphology with degenerated cristae as part of the wooden breast pathogenesis. A decrease in the number of muscle fibers and increase in the proportion of small fibers has also been documented as a characteristic of wooden breast in some broiler lines ${ }^{40}$.

A candidate gene on GGA6, GOT1, encodes the cytoplasmic version of an enzyme called aspartate transaminase (AST) that catalyzes the interconversion of aspartate and $\alpha$-ketoglutarate to oxaloacetate and glutamate. Two key roles of AST should be highlighted from this anaplerotic reaction: the replenishment of aspartate, a key intermediate of the citric acid cycle, and the regulation of concentrations of glutamate, which functions as a potentiator of insulin release and precursor of the antioxidant glutathione in human skeletal muscle ${ }^{41}$. Thus, variation in GOT1 may play an important role in altered energy metabolism and ROS balance in wooden breast.

Genetic variation associated with broiler production traits such as visceral adiposity ${ }^{42}$, body weight ${ }^{43}$ is already known to affect plasma insulin and glucagon levels, as well as insulin sensitivity and glucose clearance. More generally, commercial broilers have elevated plasma insulin levels compared to layers ${ }^{44}$ and poorer serum insulin homeostasis compared to Silky chickens ${ }^{45}$. Insulin is also a key regulator of carbohydrate, lipid, and amino acid metabolism $^{46,47}$, which are dysregulated in wooden breast and white striping ${ }^{48,49}$, although additional research is required to determine whether altered insulin dynamics are a contributing factor to wooden breast and white striping in broilers.

\section{Conclusions}

This study is the first to characterize the genetic basis of wooden breast and white striping in a commercial crossbred broiler population using genetic markers. A major finding of the present work is the identification of a QTLrich region for both wooden breast and white striping between 8.0 and 16.5 Mb on GGA5, which is homologous to and possesses highly conserved synteny with an imprinted region of human chromosome 11 . Further study of this GGA5 region may prove to be critically important for understanding the cause of chicken myopathies as well as the evolution of genomic imprinting and genes involved in growth regulation in humans. Our findings also provide compelling evidence in support of a previous hypothesis describing a shared pathomechanism between breast muscle myopathies in broilers and type 2 diabetes in mammals ${ }^{15,16}$ and suggest that potential alterations to pancreatic function and insulin action may be involved. There exist substantial differences between mammalian and avian insulin signaling and action-for example, the apparent lack of the insulin responsive glucose transporter GLUT4 in the chicken genome ${ }^{50}$ - that warrant additional research, especially with regard to wooden breast and white striping. Investigation into organs involved in regulation of whole-body energy homeostasis, such as the pancreas, liver, and adipose tissue, may also aid in our understanding of these myopathies.

\section{Methods}

Birds. All animal procedures were performed in accordance with guidelines set by The University of Delaware Institutional Animal Care and Use Committee (IACUC) and were approved by IACUC under protocol number 48R-2015-0. The study was carried out in compliance with the ARRIVE guidelines. A total of 1,228 mixed male and female Cobb500 broilers from the same breeding population of 15 sires and 200 dams were raised as two separate hatches $\left(n_{1}=686, n_{2}=542\right)$ with staggered hatch dates. Broilers were housed according to optimal industry standards in five poultry houses and given free access to feed and water until approximately 7 weeks of age (specifically 48,49,52, or 53 days), at which time they were euthanized by cervical dislocation. Live weight was recorded for all birds at 13 days of age. Preceding euthanasia (specifically 47, 48, or 52 days of age), live weight was recorded again and whole blood samples were collected from the brachial wing vein of each bird using a $3 \mathrm{~mL}$ syringe with 23-gauge needle and placed in lithium heparin-coated tubes. Plasma was separated by centrifugation and blood samples were stored at $-80^{\circ} \mathrm{C}$ until further analysis.

During necropsy, the pectoralis major muscles were evaluated for gross lesions and palpable firmness associated with wooden breast and each bird was assigned a wooden breast score using a 0-4 scale; 0-Normal indicates the bird had no macroscopic signs of the myopathy, 1-Very Mild indicates approximately $1 \%$ or less of the breast muscle was affected, 2-Mild indicates approximately between 1 and $10 \%$ of the breast muscle was affected, 3-Moderate indicates approximately between 10 and $50 \%$ of the breast muscle was affected, and a score of 4 -Severe indicates that more than $50 \%$ was affected. This scoring system was employed by Lake et al. ${ }^{16}$ in order to separate unaffected, mildly, and moderately affected chickens with higher resolution and greater sensitivity. White striping was also assessed at this time and each bird was assigned a white striping score using a $0-3$ scale; 0 -Normal indicates the bird had no macroscopic signs of white striping, 1-Mild indicates approximately $20 \%$ or less of the breast muscle was affected, 2-Moderate indicates approximately between 20 and $50 \%$ of the breast muscle was affected, and a score of 3-Severe indicates more than $50 \%$ of the muscle was affected. The incidence rates of wooden breast and white striping in males and females was compared using Pearson's Chi-squared test implemented in R with Yates' continuity correction. 
GBS library construction and DNA sequencing. After the live animal experiment was completed, blood samples from 1194 birds were selected for DNA extraction and genotyping. These represented all males $(n=557)$ and females $(n=636)$ that did not exhibit any noticeable health conditions or deformations and had a blood sample of sufficient size with no sign of coagulation. The 15 sires of our chicken population were also included for DNA extraction and genotyping to improve genotype calling of progeny; however, phenotypic data was not recorded for sires. Total DNA was isolated from blood samples using the DNeasy Blood and Tissue Kit (Qiagen) according to the manufacturer's protocol. DNA samples were quantified and quality was assessed using the NanoDrop 1000 Spectrophotometer (Thermo Fisher scientific); all samples had a 260/280 ratio of approximately 1.8 and 260/230 ratio greater than 1.5 . Approximately $2.5 \mu \mathrm{g}$ of DNA per sample was aliquoted into individual wells of a 96-well plate, dried at room temperature, sealed, and shipped to Animal Genomics Research laboratory, AgResearch Invermay, New Zealand for restriction enzyme-based genotyping by sequencing (GBS). The GBS libraries were constructed according to the methods outlined in Elshire et al. ${ }^{51}$ with modifications as in Dodds et al. ${ }^{52}$ using two restriction enzymes (MspI and ApeKI). Single-end $1 \times 100$ sequencing of 96-plex libraries was performed on an Illumina HiSeq 2500. To control and detect lane bias and batch bias, three samples of control DNA were included in each library and each library was run on at least 4 lanes across at least 2 flowcells. Additionally, one sample (B92023) was included as a positive control in all libraries.

Data filtering and SNP calling. AgResearch supplied a single variant call file (VCF) which was constructed by the following methods. Fastq files were demultiplexed using GBSX $^{53}$ and mapped by sample and lane onto the GRCg6a reference genome (Ensembl release 95) using BWA-MEM ${ }^{54}$. Samtools v1.95 ${ }^{55}$ was used to remove reads with mapping quality below 30 , convert the alignment file to BAM format, and merge all alignment files by sample. Variants were detected across all samples on a per chromosome basis using samtools and bcftools v1.9 with parameters set to limit variants to biallelic $\mathrm{SNPs}^{55}$. The results were then combined into a single VCF.

Variants were filtered to only include SNPs with a minimum read depth of 5 for at least $50 \%$ of samples, a maximum average read depth of 50 , and a minor allele frequency of $5 \%$ or greater. Samples were also filtered to only include those with a minimum read depth of 5 for at least $50 \%$ of SNPs. Loci on the W chromosome were excluded from all analyses, but were used along with $\mathrm{Z}$ chromosome loci to confirm sex of the birds before subsequent analyses. Loci on the $\mathrm{Z}$ chromosome were filtered separately using only males to avoid filtering bias from hemizygotic females.

Relatedness and genetic parameters. A genomic relationship matrix $(\boldsymbol{G})$ was constructed from the resulting variant data using the $\mathrm{R}$ package $\mathrm{KGD}^{52}$, which implements a method developed to account for GBS with low depth of coverage. Variance components and heritabilities of wooden breast, white striping, body weight at 13 days, and body weight at 7 weeks were estimated using ASReml $4^{56}$ with the following univariate model:

$$
\boldsymbol{y}=\boldsymbol{X} \boldsymbol{b}+\boldsymbol{Z} \boldsymbol{u}+e,
$$

where $y$ is a vector of phenotype values for the relevant trait, $\boldsymbol{b}$ is the vector of fixed effects and the overall mean (a vector of 1s), $\boldsymbol{X}$ is an incidence matrix for fixed effects, $\boldsymbol{u}$ is a vector of random polygenic effects, $\boldsymbol{Z}$ is an incidence matrix corresponding to $\boldsymbol{u}$, and $e$ is the residual error. Fixed effects included sex, poultry house, and age at necropsy for the phenotypes of wooden breast score and white striping score. For analysis of body weight, only sex and poultry house were included as fixed effects; although live weight at 7 weeks was measured on three separate days, this effect was disregarded because it was perfectly correlated with poultry house. Random effects $u$ and $e$ are assumed to follow normal distributions: $\boldsymbol{u} \sim N\left(0, \sigma_{g}^{2} \boldsymbol{G}\right)$ and $e \sim N\left(0, \sigma_{e}^{2} \boldsymbol{I}_{n}\right)$ where $\sigma_{g}^{2}$ is the genetic variance, $\sigma_{e}^{2}$ is the variance of the residual errors, $\boldsymbol{G}$ is the genomic relationship matrix as defined above, and $\boldsymbol{I}_{n}$ is an identity matrix of dimension $n$. Heritability was calculated as the ratio of genetic to phenotypic variance, i.e. the sum of genetic and residual variance. Pairwise genetic and phenotypic correlations between traits were estimated using bivariate models with the same fixed and random effects as specified for the univariate analyses.

Linkage disequilibrium analysis. Pairwise linkage disequilibrium (LD; $\mathrm{r}^{2}$ ) of markers within $5 \mathrm{Mb}$ of each other was calculated with Haploview v4.2 using the discrete genotype calls produced during the SNP calling step described above. To characterize LD decay with distance, markers were filtered to only include SNPs with a minimum read depth of 10 in at least $50 \%$ of birds. $\mathrm{LD}$ on the $\mathrm{Z}$ chromosome was determined using genotype calls from only male birds. LD decay curves were generated for macro-chromosomes (GGA1 through GGA5), intermediate chromosomes (GGA6 through GGA10), micro-chromosomes (GGA11 through GGA33), and the $\mathrm{Z}$ chromosome by fitting a four-parameter Weibull function (type-1) to $\mathrm{r}^{2}$ values against physical distance using the R package 'drc' (v3.0-1). The resulting curves were used to estimate $\mathrm{r}^{2}$ at distances of $1 \mathrm{~kb}, 10 \mathrm{~kb}, 100 \mathrm{~kb}$, $500 \mathrm{~kb}, 1 \mathrm{Mb}$, and $5 \mathrm{Mb}$, and to calculate the distance at which LD decayed below the threshold of $0.2\left(\mathrm{D}_{0.2}\right)$.

The ability of the present study to capture QTL was evaluated by examining SNP coverage on each chromosome in comparison to corresponding $\mathrm{D}_{0.2}$ estimates. Specifically, we calculated the percentage of each chromosome in close enough proximity to a SNP for LD to be considered useful (i.e. $\mathrm{D}_{0.2}$ ), then computed the average percentage weighted by chromosome length. This final value reflects the percentage of the genome (autosomes and $\mathrm{Z}$ chromosome) that was captured by SNPs in the present dataset.

Allele dosage estimation. Due to the relatively low-depth sequencing methods implemented here, continuous genotype probabilities were used for all association analyses rather than discrete genotype calls. Geno- 
type posterior probabilities were calculated using the Bayesian genotype caller polyRAD v1.1 ${ }^{57}$, with population structure and LD used as a prior and default parameter values. The minimum correlation coefficient between two alleles $\left(r^{2}\right)$ for LD to be used in genotype estimation was set at 0.2 and the distance within which to search for alleles that may be in LD with the given allele was set to 10,000 base pairs. The maximum mean difference in allele frequencies between iterations to be tolerated before iterations end was set to 0.001 . The resulting genotype posterior probabilities were converted to allele dosages (i.e. posterior mean genotypes) for use in association analysis. Genotype calling of markers on the $\mathrm{Z}$ chromosome was performed using only male birds.

Genome-wide association analyses. Two separate approaches-Bayesian multi-marker regression and single-SNP analysis-were used to detect quantitative trait loci (QTL) for each trait. Bayesian models were implemented in GenSel ${ }^{58}$ and involved first estimating the proportion of markers with null effect $(\pi)$ using BayesC $\pi$, followed by computation of the genetic variance explained by each $1 \mathrm{Mb}$ window of SNPs in the genome with BayesB. The BayesC $\pi$ algorithm was run for 200,000 MCMC iterations with a burn-in of 150,000 iterations and used a starting value of 0.5 for $\pi$. BayesB was run for 60,000 iterations with a burn-in of 20,000 and used the posterior mean of $\pi$ calculated by BayesC $\pi$ for each trait. The following model was used for both Bayesian methods:

$$
\boldsymbol{y}=\boldsymbol{X} \boldsymbol{b}+\sum_{k=1}^{K} \boldsymbol{z}_{k} a_{k}+e,
$$

where $K$ is the number of SNPs, $z_{k}$ is a vector of allele dosages at SNP $k, a_{k}$ is the additive effect of that SNP, and the remaining variables are as previously described. Loci on the $\mathrm{Z}$ chromosome were disregarded for this analysis. Windows explaining greater than $1 \%$ of genetic variation were considered significant and those explaining greater than $0.5 \%$ were considered suggestive.

Single-SNP analysis was performed with a mixed linear model using GCTA version $1.26 .0^{59}$ with software patches provided by van den Berg et al. ${ }^{60}$ that adapted the program to accept allele dosages. Equation 1 was used for all single-SNP analyses with the addition of a SNP effect fitted as a fixed covariate. Single-SNP analysis was performed three separate times for each trait, once using all birds, once with only females, and once with only males. Loci on the $Z$ chromosome were only included when male birds were analyzed separately. For analyses including only male or female progeny, the fixed effect of sex was dropped from the model. Significant and suggestive genome-wide association thresholds were set at $\mathrm{p}$-values corresponding to FDR-adjusted ${ }^{61} \mathrm{q}$-values of 0.05 and 0.20 , respectively.

Significant intra-chromosomal SNPs that were in high LD $\left(\mathrm{r}^{2}>0.75\right)$ or that were separated by a distance less than or equal to the $\mathrm{D}_{0.2}$ value for the relevant chromosome size were assumed to share evidence in association analyses for a given trait and were thus grouped into non-overlapping QTL. Markers considered "suggestive" were not used in the construction of QTL. Candidate genes were identified based on proximity to QTL (within 5000 bp up- or downstream of the start or end position of a QTL, respectively) using Ensembl and NCBI annotation of the Gallus gallus genome version GRCg6a (annotation release 99).

Comparative genomics. An $\sim 8 \mathrm{Mb}$ region of GGA5 was found to be exceedingly rich in QTL for both wooden breast and white striping, and warranted additional attention with regard to existing knowledge of the chromosome's genomic features, which were visualized in a Circos $\operatorname{plot}^{62}$. To this end, sequence alignments between chicken genome assembly GRCg6a and human genome assembly GRCh38 (vertebrate net alignment netHg38) and between human genome assembly GRCh38 and mouse genome assembly GRCm38 (placental net alignment netMm10) were retrieved using the UCSC Table Browser ${ }^{63}$. Associations with human muscular and metabolic disease phenotypes in genomic regions homologous to the QTL-rich section of GGA5 were identified from the OMIM database ${ }^{64}$. Additionally, previously identified selective sweeps on GGA5 in commercial purebred broiler lines that are susceptible to wooden breast ${ }^{33}$ were lifted over to the GRCg6a chicken genome assembly using the UCSC liftOver tool ${ }^{65}$.

\section{Data availability}

The datasets generated during the current study are available in the NCBI Sequence Read Archive (SRA) repository under BioProject PRJNA682423.

Received: 10 December 2020; Accepted: 8 March 2021

Published online: 24 March 2021

\section{References}

1. Petracci, M., Mudalal, S., Soglia, F. \& Cavani, C. Meat quality in fast-growing broiler chickens. Worlds. Poult. Sci. J. 71, 363-374 (2015).

2. Havenstein, G. B., Ferket, P. R. \& Qureshi, M. A. Growth, livability, and feed conversion of 1957 versus 2001 broilers when fed representative 1957 an 2001 broiler diets. Poult. Sci. 82, 1500-1508 (2003).

3. Havenstein, G. B., Ferket, P. R. \& Qureshi, M. A. Carcass composition and yield of 1957 versus 2001 broilers when fed representative 1957 and 2001 broiler diets. Poult. Sci. 82, 1509-1518 (2003).

4. Sihvo, H. K., Immonen, K. \& Puolanne, E. Myodegeneration with fibrosis and regeneration in the pectoralis major muscle of broilers. Vet. Pathol. 51, 619-623 (2014).

5. Papah, M. B., Brannick, E. M., Schmidt, C. J. \& Abasht, B. Evidence and role of phlebitis and lipid infiltration in the onset and pathogenesis of Wooden Breast Disease in modern broiler chickens. Avian Pathol. 46, 623-643 (2017). 
6. Norring, M. et al. Wooden breast myopathy links with poorer gait in broiler chickens. Animal https://doi.org/10.1017/S175173111 8003270 (2018).

7. Griffin, J. R., Moraes, L., Wick, M. \& Lilburn, M. S. Onset of white striping and progression into wooden breast as defined by myopathic changes underlying Pectoralis major growth. Estimation of growth parameters as predictors for stage of myopathy progression. Avian Pathol. 47, 2-13 (2018).

8. Mutryn, M. F., Brannick, E. M., Fu, W., Lee, W. R. \& Abasht, B. Characterization of a novel chicken muscle disorder through differential gene expression and pathway analysis using RNA-sequencing. BMC Genom. 16, 399 (2015).

9. Kuttappan, V. A. et al. Pathological changes associated with white striping in broiler breast muscles. Poult. Sci. 92, 331-338 (2013).

10. Livingston, M. L., Landon, C. D., Barnes, H. J., Brake, J. \& Livingston, K. A. Dietary potassium and available phosphorous on broiler growth performance, carcass characteristics, and wooden breast. Poult. Sci. 98, 2813-2822 (2019).

11. Zampiga, M., Soglia, F., Petracci, M., Meluzzi, A. \& Sirri, F. Effect of different arginine-to-lysine ratios in broiler chicken diets on the occurrence of breast myopathies and meat quality attributes. Poult. Sci. 98, 2691-2697 (2019).

12. Meloche, K. J., Fancher, B. I., Emmerson, D. A., Bilgili, S. F. \& Dozier, W. A. Effects of reduced dietary energy and amino acid density on Pectoralis major myopathies in broiler chickens at 36 and 49 days of age1. Poult. Sci. 97, 1794-1807 (2018).

13. Soglia, F., Mazzoni, M. \& Petracci, M. Spotlight on avian pathology: current growth-related breast meat abnormalities in broilers. Avian Pathol. 48, 1-3 (2019).

14. Boerboom, G., van Kempen, T., Navarro-villa, A. \& Perez-Bonilla, A. Unraveling the cause of white striping in broilers using metabolomics. Poult. Sci. 97, 3977-3986 (2018).

15. Lake, J. A. \& Abasht, B. Glucolipotoxicity: a proposed etiology for wooden breast and related myopathies in commercial broiler chickens. Front. Physiol. https://doi.org/10.3389/fphys.2020.00169 (2020).

16. Lake, J. A., Papah, M. B. \& Abasht, B. Increased expression of lipid metabolism genes in early stages of wooden breast links myopathy of broilers to metabolic syndrome in humans. Genes (Basel) 10, 746 (2019).

17. Bailey, R. A., Souza, E. \& Avendano, S. Characterising the influence of genetics on breast muscle myopathies in broiler chickens. Front. Physiol. 11, 1-12 (2020).

18. Alnahhas, N. et al. Genetic parameters of white striping in relation to body weight, carcass composition, and meat quality traits in two broiler lines divergently selected for the ultimate $\mathrm{pH}$ of the pectoralis major muscle. BMC Genet. 17, 1-9 (2016).

19. Pampouille, E. et al. Mapping QTL for white striping in relation to breast muscle yield and meat quality traits in broiler chickens. BMC Genom. 19, 1-14 (2018).

20. Bailey, R. A., Watson, K. A., Bilgili, S. F. \& Avendano, S. The genetic basis of pectoralis major myopathies in modern broiler chicken lines. Poult. Sci. 94, 2870-2879 (2015).

21. Meloche, K. J., Fancher, B. I., Emmerson, D. A., Bilgili, S. F. \& Dozier, W. A. Effects of quantitative nutrient allocation on myopathies of the Pectoralis major muscles in broiler chickens at 32, 43, and 50 days of age 1. Poult. Sci. 97, 1786-1793 (2018).

22. Chan, A. W., Hamblin, M. T. \& Jannink, J. L. Evaluating imputation algorithms for low-depth genotyping-by-sequencing (GBS) data. PLoS ONE 11, 1-17 (2016).

23. Travers, M. E. et al. Insights into the molecular mechanism for type 2 diabetes susceptibility at the KCNQ1 locus from temporal changes in imprinting status in human islets. Diabetes 62, 987-992 (2013).

24. Fan, F. et al. Dynamin 2 regulates biphasic insulin secretion and plasma glucose homeostasis. J. Clin. Invest. 125, 4026-4041 (2015).

25. Xu, Z., Wei, G., Chepelev, I., Zhao, K. \& Felsenfeld, G. Mapping of INS promoter interactions reveals its role in long-range regulation of SYT8 transcription. Nat. Struct. Mol. Biol. 18, 372-378 (2011).

26. Mutair, A. N. A. et al. Heterogeneity in phenotype of usher-congenital hyperinsulinism syndrome. Diabetes Care 36, 557-561 (2013).

27. Mohlke, K. L. \& Boehnke, M. Recent advances in understanding the genetic architecture of type 2 diabetes. Hum. Mol. Genet. 24, R85-R92 (2015).

28. Kimber, E., Tajsharghi, H., Kroksmark, A. K., Oldfors, A. \& Tulinius, M. A mutation in the fast skeletal muscle troponin I gene causes myopathy and distal arthrogryposis. Neurology 67, 597-601 (2006).

29. Liu, L. et al. LSP1 is an endothelial gatekeeper of leukocyte transendothelial migration. J. Exp. Med. 201, 409-418 (2005).

30. Pitel, F. et al. A high-resolution radiation hybrid map of chicken chromosome 5 and comparison with human chromosomes. BMC Genom. 5, 1-9 (2004).

31. Smith, A. C., Choufani, S., Ferreira, J. C. \& Weksberg, R. Growth regulation, imprinted genes, and chromosome 11p15.5. Pediatr. Res. 61, 43-47 (2007).

32. Paulsen, M., Khare, T., Burgard, C., Tierling, S. \& Walter, J. Evolution of the Beckwith-Wiedemann syndrome region in vertebrates. Genome Res. 15, 146-153 (2005).

33. Fu, W., Lee, W. R. \& Abasht, B. Detection of genomic signatures of recent selection in commercial broiler chickens. BMC Genet. $17,1-10(2016)$.

34. Abbott, G. W. Biology of the KCNQ1 potassium channel. New J. Sci. 2014, 1-26 (2014).

35. Torekov, S. S. et al. KCNQ1 long QT syndrome patients have hyperinsulinemia and symptomatic hypoglycemia. Diabetes 63, $1315-1325(2014)$

36. Gall, S. et al. Wooden breast in commercial broilers associated with mortality, dorsal recumbency, and pulmonary disease. Avian Dis. 63, 514-519 (2019).

37. Romero, N. B. Centronuclear myopathies: a widening concept. Neuromuscul. Disord. 20, 223-228 (2010).

38. Fraysse, B., Guicheney, P. \& Bitoun, M. Calcium homeostasis alterations in a mouse model of the dynamin 2-related centronuclear myopathy. Biol. Open 5, 1691-1696 (2016).

39. Tinelli, E., Pereira, J. A. \& Suter, U. Muscle-specific function of the centronuclear myopathy and charcot-marie-tooth neuropathy associated dynamin 2 is required for proper lipid metabolism, mitochondria, muscle fibers, neuromuscular junctions and peripheral nerves. Hum. Mol. Genet. 22, 4417-4429 (2013).

40. Velleman, S. G., Clark, D. L. \& Tonniges, J. R. The effect of the Wooden Breast myopathy on sarcomere structure and organization. Avian Dis. 62, 28-35 (2018).

41. Rutten, E. P. A., Engelen, M. P. K. J., Schols, A. M. W. J. \& Deutz, N. E. P. Skeletal muscle glutamate metabolism in health and disease: state of the art. Curr. Opin. Clin. Nutr. Metab. Care 8, 41-51 (2005).

42. Simon, J. \& LeClercq, B. Longitudinal study of adiposity in chickens selected for high or low abdominal fat content: further evidence of a glucose-insulin imbalance in the fat line. J. Nutr. 112, 1961-1973 (1982).

43. Sinsigalli, N. A., Mcmurtry, J. P., Cherry, J. A. \& Siegel, P. B. Glucose tolerance, plasma insulin and immunoreactive glucagon in chickens selected for high and low body weight. J. Nutr. 8, 941-947 (1987).

44. Shiraishi, J. I. et al. Comparisons of insulin related parameters in commercial-type chicks: evidence for insulin resistance in broiler chicks. Physiol. Behav. 103, 233-239 (2011).

45. Ji, J. et al. Dynamic changes of blood glucose, serum biochemical parameters and gene expression in response to exogenous insulin in Arbor Acres broilers and Silky fowls. Sci. Rep. 10, 1-11 (2020).

46. Tokushima, Y., Takahashi, K., Sato, K. \& Akiba, Y. Glucose uptake in vivo in skeletal muscles of insulin-injected chicks. Comp. Biochem. Physiol. B Biochem. Mol. Biol. 141, 43-48 (2005).

47. Godet, E. et al. Insulin immuno-neutralization in chicken: effects on insulin signaling and gene expression in liver and muscle. J. Endocrinol. 197, 531-542 (2008). 
48. Abasht, B., Mutryn, M. F., Michalek, R. D. \& Lee, W. R. Oxidative stress and metabolic perturbations in wooden breast disorder in chickens. PLoS ONE 11, 1-16 (2016).

49. Papah, M. B. \& Abasht, B. Dysregulation of lipid metabolism and appearance of slow myofiber- specific isoforms accompany the development of Wooden Breast myopathy in modern broiler chickens. Sci. Rep. 9, 17170 (2019).

50. Seki, Y., Sato, K., Kono, T., Abe, H. \& Akiba, Y. Broiler chickens (Ross strain) lack insulin-responsive glucose transporter GLUT4 and have GLUT8 cDNA. Gen. Comp. Endocrinol. 133, 80-87 (2003).

51. Elshire, R. J. et al. A robust, simple genotyping-by-sequencing (GBS) approach for high diversity species. PLoS ONE 6, e19379 (2011).

52. Dodds, K. G. et al. Construction of relatedness matrices using genotyping-by-sequencing data. BMC Genom. 16, 1-15 (2015).

53. Herten, K., Hestand, M. S., Vermeesch, J. R. \& Van Houdt, J. K. J. GBSX: a toolkit for experimental design and demultiplexing genotyping by sequencing experiments. BMC Bioinform. 16, 1-6 (2015).

54. Li, H. Aligning sequence reads, clone sequences and assembly contigs with BWA-MEM. arXiv Prepr. (2013).

55. Li, H. A statistical framework for SNP calling, mutation discovery, association mapping and population genetical parameter estimation from sequencing data. Bioinformatics 27, 2987-2993 (2011).

56. Butler, D. G., Cullis, B. R., Gilmour, A., Gogel, B. J. \& Thompson, R. ASReml-R Reference Manual Version 4. (2018).

57. Clark, L. V., Lipka, A. E. \& Sacks, E. J. polyRAD: genotype calling with uncertainty from sequencing data in polyploids and diploids. Genes Genom. Genet. 9, 663-673 (2019).

58. Fernando, R. L. \& Garrick, D. J. GenSel-User manual for a portfolio of genomic selection related analyses. in Iowa State University 0-24 (2009).

59. Yang, J., Lee, S. H., Goddard, M. E. \& Visscher, P. M. GCTA: a tool for genome-wide complex trait analysis. Am. J. Hum. Genet. 88, 76-82 (2011).

60. Van Den Berg, S. et al. Imputation to whole-genome sequence using multiple pig populations and its use in genome-wide association studies. Genet. Sel. Evol. 51, 1-13 (2019).

61. Benjamini, Y. \& Hochberg, Y. Controlling the false discovery rate : a practical and powerful approach to multiple testing. J. R. Stat. Soc. Ser. B 57, 289-300 (1995).

62. Krzywinski, M. I. et al. Circos: an information aesthetic for comparative genomics. Genome Res. 19, 1639-1645 (2009).

63. Karolchik, D. et al. The UCSC table browser data retrieval tool. Nucleic Acids Res. D https://doi.org/10.1093/nar/gkh103 (2004).

64. Online Mendelian Inheritance in Man, OMIM. McKusick-Nathans Institute of Genetic Medicine, Johns Hopkins University (Baltimore, MD) Available at: https://omim.org/. (Accessed: 14th April 2020).

65. Hinrichs, A. et al. The UCSC genome browser database: update 2006. Nucleic Acids Res. 1, D590-D598 (2006).

\section{Acknowledgements}

We would like to thank Muhammed Walugembe, Luke Kramer, and numerous others from the Department of Animal Science at Iowa State University for their excellent technical assistance. Andrew Hess of AgResearch proved indispensable in providing guidance on analytical methods specific to low-depth GBS and Rudiger Brauning of AgResearch was always immensely helpful and relentlessly cheerful in the face of numerous questions throughout the entirety of this project. We gratefully acknowledge the in-kind support by Cobb-Vantress Inc. in providing chicks and chicken feed for this experiment. We also greatly appreciate assistance with samples and data collection from many graduate and undergraduate students at the University of Delaware Department of Animal and Food Sciences. We would like to acknowledge the support from the University of Delaware Center for Bioinformatics and Computational Biology for utilization of their cluster BioMix, which was made possible through support from the Delaware INBRE (NIH GM103446), the state of Delaware and the Delaware Biotechnology Institute (DBI). RNA-sequencing services at DBI are greatly appreciated.

\section{Author contributions}

J.A.L. analyzed the data and wrote the manuscript. B.A. conceived of the study, supervised the study and analysis, and contributed to interpretation of results and manuscript revision. J.C.M.D. supervised the statistical analysis and revised the manuscript. All authors reviewed the manuscript.

\section{Competing interests}

The authors declare no competing interests.

\section{Additional information}

Supplementary Information The online version contains supplementary material available at https://doi.org/ 10.1038/s41598-021-86176-4.

Correspondence and requests for materials should be addressed to B.A.

Reprints and permissions information is available at www.nature.com/reprints.

Publisher's note Springer Nature remains neutral with regard to jurisdictional claims in published maps and institutional affiliations.

(c) (1) Open Access This article is licensed under a Creative Commons Attribution 4.0 International License, which permits use, sharing, adaptation, distribution and reproduction in any medium or format, as long as you give appropriate credit to the original author(s) and the source, provide a link to the Creative Commons licence, and indicate if changes were made. The images or other third party material in this article are included in the article's Creative Commons licence, unless indicated otherwise in a credit line to the material. If material is not included in the article's Creative Commons licence and your intended use is not permitted by statutory regulation or exceeds the permitted use, you will need to obtain permission directly from the copyright holder. To view a copy of this licence, visit http://creativecommons.org/licenses/by/4.0/.

(C) The Author(s) 2021 